\title{
Geranylgeranylacetone alleviates radiation-induced lung injury by inhibiting epithelial-to-mesenchymal transition signaling
}

\author{
JOONG-SUN KIM ${ }^{1}$, YEONGHOON SON ${ }^{2}$, MYUNG-GU JUNG ${ }^{2}$, YE JI JEONG ${ }^{2}$, \\ SUNG-HO KIM ${ }^{3}$, SU-JAE LEE ${ }^{4}$, YOON-JIN LEE ${ }^{2}$ and HAE-JUNE LEE ${ }^{2}$
}

\begin{abstract}
${ }^{1}$ Research Center, Dongnam Institute of Radiological and Medical Sciences, Busan 619-953; ${ }^{2}$ Division of Radiation Effects, Korea Institute of Radiological and Medical Sciences, Seoul 139-706; ${ }^{3}$ Department of Veterinary Anatomy, College of Veterinary Medicine, Chonnam National University, Gwangju 500-757; ${ }^{4}$ Laboratory of Molecular Biochemistry, Department of Life Science, Research Institute for Natural Sciences, Hanyang University, Seoul 133-791, Republic of Korea
\end{abstract}

Received May 4, 2015; Accepted March 9, 2016

DOI: $10.3892 / \mathrm{mmr} .2016 .5121$

\begin{abstract}
Radiation-induced lung injury (RILI) involves pneumonitis and fibrosis, and results in pulmonary dysfunction. Moreover, RILI can be a fatal complication of thoracic radiotherapy. The present study investigated the protective effect of geranylgeranlyacetone (GGA), an inducer of heat shock protein (HSP)70, on RILI using a C57BL/6 mouse model of RILI developing 6 months subsequent to exposure to $12.5 \mathrm{~Gy}$ thoracic radiation. GGA was administered 5 times orally prior and subsequent to radiation exposure, and the results were assessed by histological analysis and western blotting. The results show that late RILI was alleviated by GGA treatment, possibly through the suppression of epithelial-to-mesenchymal transition (EMT) marker expression. Based on histological examination, orally administered GGA during the acute phase of radiation injury not only significantly inhibited pro-surfactant protein C (pro-SPC) and vimentin expression, but also preserved E-cadherin expression 6 months after irradiation-induced injury of the lungs. GGA induced HSP70 and inhibited EMT marker expression in L132 human lung epithelial cells following IR. These data suggest that the prevention of EMT signaling is a key cytoprotective effect in the context of RILI. Thus, HSP70-inducing drugs, such as GGA, could be beneficial for protection against RILI.
\end{abstract}

\section{Introduction}

Radiotherapy is an essential and common treatment modality used for various malignancies located within the thoracic

Correspondence to: Dr Hae-June Lee or Dr Yoon-Jin Lee, Division of Radiation Effects, Korea Institute of Radiological and Medical Sciences, 75 Nowongil, Nowon-Gu, Seoul 139-706, Republic of Korea

E-mail: hjlee@kirams.re.kr

E-mail: yjlee8@kirams.re.kr

Key words: geranylgeranylacetone, heat shock protein 70, thoracic radiation, lung fibrosis, epithelial to mesenchymal transition region. Thoracic radiotherapy is limited by toxicity to normal lung tissue in a radiation volume- and dose-dependent manner (1). Clinically, $10-30 \%$ of patients with cancer in the thoracic region suffer radiation-induced lung injury (RILI) $(2,3)$. Thus, alleviating RILI could improve the efficacy of cancer treatment and the quality of life for the patient.

RILI is a complex pathological process that results in early pneumonitis and late pulmonary fibrosis (4). Radiation to the thorax causes pulmonary fibrosis by releasing pro-inflammatory cytokines and activating myofibroblasts, which produce collagen and extracellular matrix (5). Alveolar epithelial cells participate in the pathogenesis of pulmonary fibrosis by producing pro-inflammatory mediators and undergoing epithelial-to-mesenchymal transition (EMT) (6). Recent studies have suggested that epithelial cells undergo morphological changes to acquire fibroblast or myofibroblast markers, such as $\alpha$-smooth muscle actin ( $\alpha$ SMA), $\mathrm{N}$-cadherin and vimentin, while showing decreased expression of epithelial markers, such as E-cadherin (7-9).

Geranylgeranlyacetone (GGA) is a nontoxic anti-ulcer drug that has been reported to induce heat shock protein (HSP)70 (10,11). HSP70 has a cytoprotective property as an intracellular chaperone by modulating the immune response and maintaining cellular homeostasis (12-14). GGA treatment has been shown to protect against various diseases in animal models and humans via HSP70 induction $(15,16)$. It was reported that HSP70 may inhibit EMT by inhibiting the generation of reactive oxygen species (17).

The aim of the present study was to investigate the protective effect of GGA on RILI via inhibiting radiation-induced EMT and to provide mechanistic insights into the development of pharmacological therapeutics to treat or mitigate RILI.

\section{Materials and methods}

Animals and GGA treatments. All protocols in this study were approved by the Institutional Animal Care and Use Committee of the Korean Institute of Radiological and Medical Sciences (Seoul, Korea; IACUC permit number: KIRAMS2013-035). Seven-week-old female C57BL/6 mice $\sim 20 \mathrm{~g}$ were obtained from Orient Bio Co. Ltd. (Sungnam, 
Korea). The mice were housed for 1 week prior to conducting the experiments and were randomly assigned to the following three groups: i) Non-irradiated control $(n=7)$; ii) thoracic irradiation (IR) control $(n=9)$; and iii) GGA+IR $(n=9)$. The animals were housed at $20 \pm 2^{\circ} \mathrm{C}$ with $50 \pm 10 \%$ humidity with a $12 / 12 \mathrm{~h}$ light/dark cycle in a specific pathogen-free facility and were fed a normal diet and autoclaved water ad libitum.

GGA was obtained from Santa Cruz Biotechnology, Inc. (Santa Cruz, CA, USA). GGA (200 mg/kg) was orally administered 24 and $1 \mathrm{~h}$ prior to IR treatment and 24, 48 and $72 \mathrm{~h}$ after IR treatment. Each mouse assigned to the radiation treatment group was exposed to radiation under anesthesia $[30 \mathrm{mg} / \mathrm{kg}$ Zoletil (Virbac, Carros, France) and $10 \mathrm{mg} / \mathrm{kg}$ Rompun (Bayer AG, Leverkusen, Germany)] using an X-Rad320 (Precision X-Ray, East Haven, CT, USA; filter, $2 \mathrm{~mm}$; aluminium, $42 \mathrm{~cm}$, $260 \mathrm{kV} / \mathrm{s}, 10 \mathrm{~mA}, 2.0 \mathrm{~Gy} / \mathrm{min})$. The radiation field size was $20 \times 50 \mathrm{~mm}$ for the thoracic irradiation of the mice. The mice were euthanized 6 months after IR by inhalation of $\mathrm{CO}_{2}$.

Cell culture and treatments. The L132 human lung epithelial cell line (American Type Culture Collection, Manassas, VA, USA) was cultured in Dulbecco's modified Eagle's medium (Welgene, Inc., Gyeongsangbuk-do, Korea) with $10 \%$ fetal bovine serum (Corning Incorporated, Corning, NY, USA) and antibiotics (Gibco; Thermo Fisher Scientific, Inc., Waltham, $\mathrm{MA}, \mathrm{USA}$ ) at $37^{\circ} \mathrm{C}$ in the presence of $5 \% \mathrm{CO}_{2}$. GGA was added to the culture medium for $24 \mathrm{~h}$ before IR treatment.

Lung tissue preparation. Six months after IR, all of the mice from the three groups were euthanized. The lungs of 3 mice from each group were injected with $1 \mathrm{ml}$ neutralized formalin (Yakuri Pure Chemicals Co., Ltd., Kyoto, Japan) and then fixed in formalin for $48 \mathrm{~h}$. The whole lungs of 3 mice from each group were injected with $1 \mathrm{ml}$ neutralized formalin, fixed in $10 \%$ formalin solution for $48 \mathrm{~h}$ and then were embedded in paraffin for the histological examination. The left lobes of the lungs ( $n=4$ per control group, and $n=6$ per IR and GGA+IR group) were resected and fixed in formalin for histological analysis, and the right lobes were frozen in liquid nitrogen and stored at $-80^{\circ} \mathrm{C}$ for western blotting.

Histopathology and immunohistochemical staining. Paraffin-embedded sections were stained with hematoxylin and eosin (H\&E) or with Masson's Trichrome staining kit (Sigma-Aldrich, St. Louis, MO, USA). Immunohistochemistry was conducted using a Vectastain Elite ABC kit (Vector Laboratories Inc., Burlingame, CA, USA) based on the manufacturer's protocol. For antigen retrieval, the sections were boiled in citrate buffer $\left(\mathrm{pH} 6.0 ; 95-98^{\circ} \mathrm{C}\right)$ in a water bath for $30 \mathrm{~min}$ followed by cooling for $20 \mathrm{~min}$ at room temperature. The sections were then incubated overnight at $4^{\circ} \mathrm{C}$ with the following primary antibodies: Mouse monoclonal anti-8-hydroxy-2'-deoxyguanosine (8-OHdG) (sc-66036; 1:100; Santa Cruz Biotechnology, Inc.), rabbit polyclonal anti-pro-SPC (AB3786; 1:500; Millipore, Darmstadt, Germany), mouse monoclonal anti-E-cadherin (sc-8426; 1:100; Santa Cruz Biotechnology, Inc.) and mouse monoclonal anti-vimentin (sc-6260; 1:100; Santa Cruz Biotechnology, Inc.), after which the slides were washed with phosphate-buffered saline (PBS) containing $0.05 \%$ Triton X-100 (Sigma-Aldrich). Then, the sections were incubated with the corresponding secondary antibody for $30 \mathrm{~min}$ and counterstained with hematoxylin (Sigma-Aldrich). For immunofluorescent staining, sections stained with primary antibodies were incubated with the appropriate fluorescently-labeled secondary antibodies (1:250; Molecular Probes Inc., Eugene, OR, USA) and then counterstained with 4,6-diamidino-2-phenylindole dihydrochloride (DAPI; $3 \mu \mathrm{M}$; Sigma-Aldrich). Images obtained using an Olympus BX53 microscope (Olympus Corporation, Tokyo, Japan) equipped with a CCD camera (ProgRes CF Scan; Jenoptik, Jena, Germany).

Western blot analysis. Lung tissues were physically minced, then proteins were extracted from the lysates using Pro-prep solution (17081; Intron Inc., Sungnam, Korea). The concentration of total protein was quantified using Bio-Rad Laboratories Protein Assay kit II (Bio-Rad Laboratories, Inc., Hercules, CA, USA). Proteins were separated by electrophoresis on a 6-12.5\% sodium dodecyl sulfate-polyacrylamide gel and were then transferred onto $0.45 \mu \mathrm{m}$ nitrocellulose membranes (Pall Corporation, Willoughby, $\mathrm{OH}, \mathrm{USA}$ ) for $2 \mathrm{~h}$ at $100 \mathrm{~V}$. The membranes were blocked with 5\% non-fat dry milk in PBS containing $0.1 \%$ Tween-20 (Sigma-Aldrich) for $30 \mathrm{~min}$. The membranes were subsequently incubated overnight at $4^{\circ} \mathrm{C}$ with the following antibodies: Mouse monoclonal anti-HSP70 (ADI-SPA-810; 1:1,000; Enzo Life Sciences, Farmingdale, NY, USA), mouse monoclonal anti-E-cadherin (sc-8426; 1:1,000; Santa Cruz Biotechnology, Inc.), mouse monoclonal anti-vimentin (sc-6260; 1:1,000; Santa Cruz), mouse monoclonal anti-N-cadherin (610920; 1:1,000; BD Biosciences, Franklin Lakes, NJ, USA) and mouse monoclonal $\beta$-actin (A1978; 1:3,000; Sigma-Aldrich). Then membranes were then washed thoroughly in PBS-0.1\% Tween-20 and incubated for $1 \mathrm{~h}$ with the following horseradish-conjugated secondary antibodies from Santa Cruz Biotechnology, Inc. at a dilution of 1:3,000: Goat anti-mouse IgG (sc-2005), donkey anti-goat IgG (sc-2020), goat anti-rabbit IgG (sc-2004). Protein bands were visualized by electrochemiluminescence (G:BOX Chemi XT6; Syngene, Cambridge, UK). Protein expression was then quantified using a Fluor-S MultiImager (Bio-Rad Laboratories, Inc.).

Statistical analysis. Data are presented as the mean \pm standard deviation for each experiment. Statistical analysis was performed using one-way analysis of variance and the Tukey post-hoc test for multiple comparisons with GraphPad Prism version 6.0 software (GraphPad Software, Inc., La Jolla, CA, USA). P<0.05 was considered to indicate a statistically significant difference.

\section{Results}

GGA alleviates radiation-induced lung injury in late phase. At 24 weeks post-IR, Masson's trichrome staining showed that the IR group experienced marked fibrotic changes and collagen accumulation in the lung parenchyma. GGA treatment markedly alleviated late phase RILI, which was reflected by the reduction in pathological changes to the lung parenchyma (Fig. 1A). Immunoblotting data showed increased expression of collagen I and $\alpha$ SMA in the IR group when compared with the control-treated mice. GGA treatment significantly prevented expression of collagen I and $\alpha$ SMA in the lungs associated with IR $(\mathrm{P}<0.05$, Fig. 1B). 

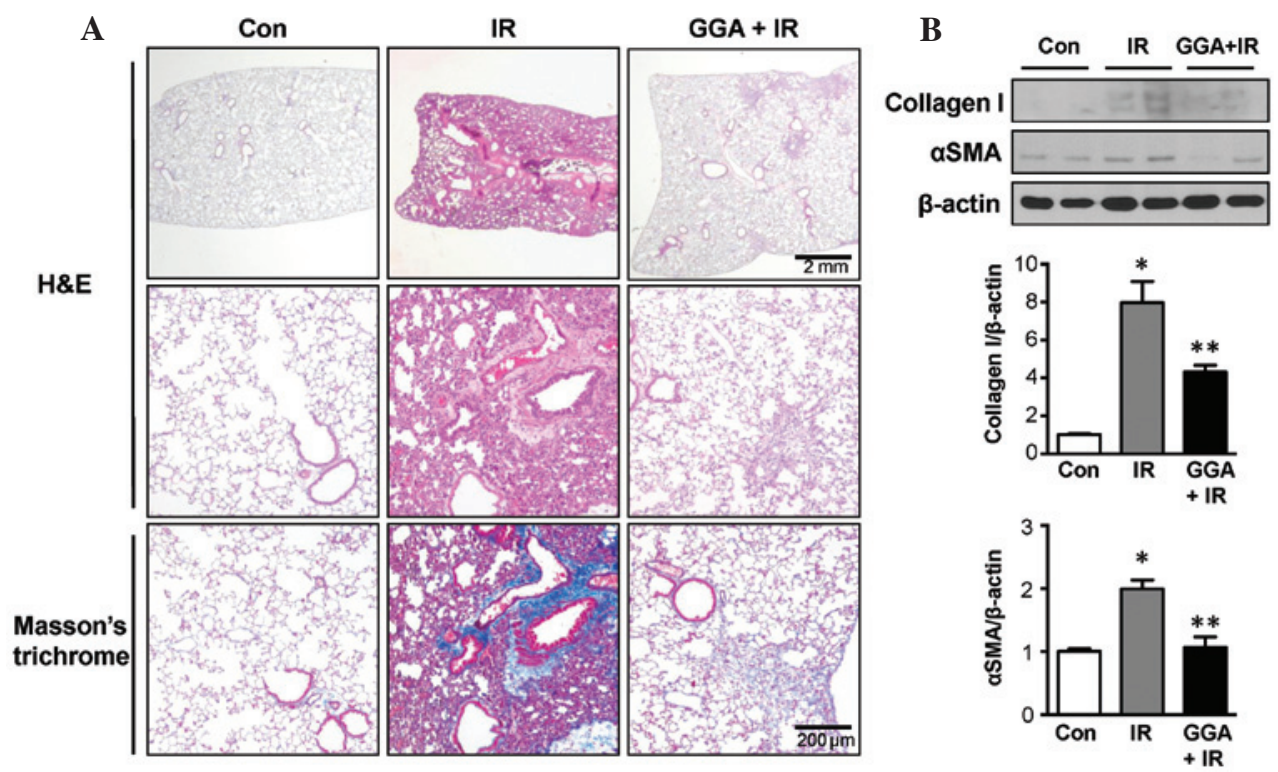

Figure 1. A 12.5-Gy dose of thoracic IR to mouse lungs leads to pneumonitis and regional fibrosis. (A) C57BL/6 mice received thoracic radiation, after which the lungs were harvested and subjected to hematoxylin and eosin staining and Masson's trichrome staining 6 months after IR. Representative images show the lungs of the non-IR control, IR and GGA+IR groups. (B) Representative western blotting data for collagen I and $\alpha$ SMA expression are shown and quantified. A statistically significant difference ( $\mathrm{n}=5$ mice per group; ${ }^{*} \mathrm{P}<0.05$ vs. control, ${ }^{* *} \mathrm{P}<0.05$ vs. IR control) was observed in collagen I and $\alpha \mathrm{SMA}$ expression in the lungs harvested from mice treated with IR and GGA. IR, irradiation; GGA, geranylgeranylacetone; $\alpha$ SMA, $\alpha$ smooth muscle actin.
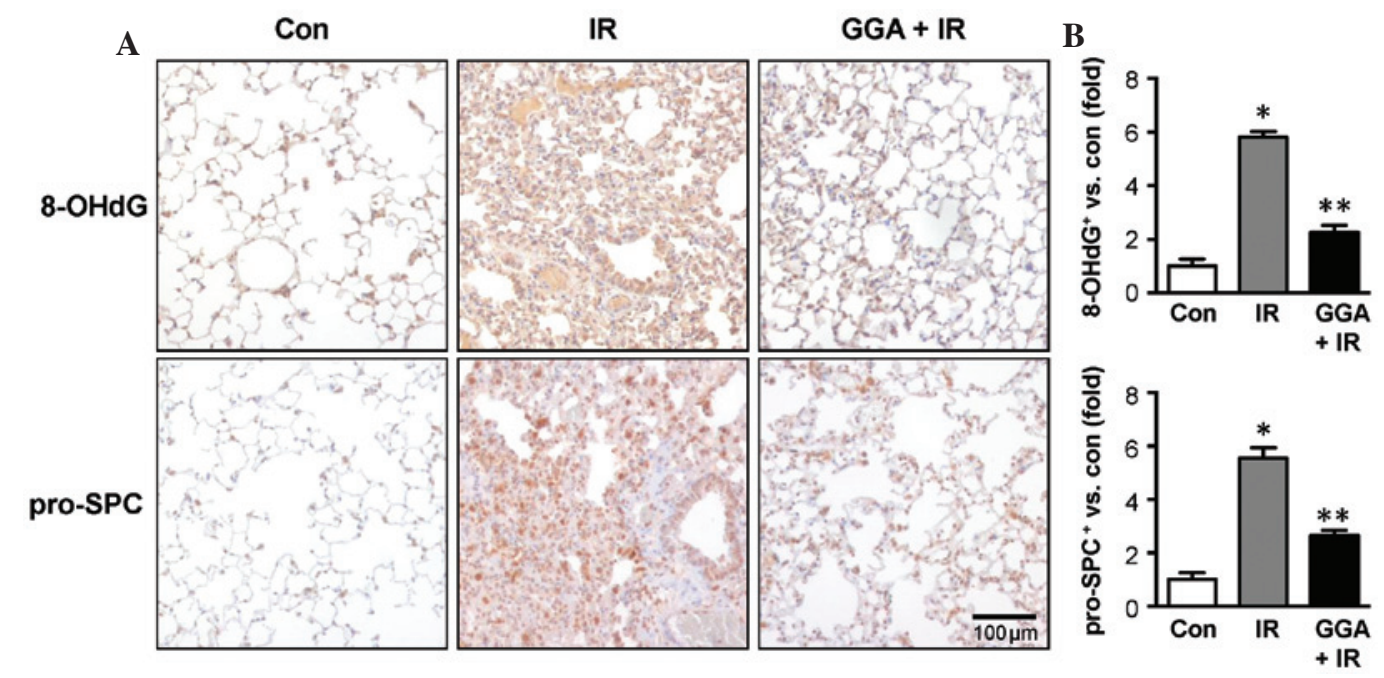

Figure 2. Evaluation of the cytoprotective effect of GGA on IR-induced mouse lung injury. (A) Representative images of 8-OHdG and pro-SPC expression in the lungs by immunohistochemistry. (B) Graphs show the quantification of the area of positive staining presented as the mean \pm standard deviation (n=3 per group; ${ }^{*} \mathrm{P}<0.05$ vs. control, ${ }^{* *} \mathrm{P}<0.05$ vs. IR control). GGA, geranylgeranylacetone; IR, irradiation; 8-OHdG, 8-hydroxy-2'-deoxyguanosine; SPC, surfactant protein C.

GGA protects lung epithelial cells against IR. Twenty-four weeks after treatment, the IR group showed high expression of 8 -OHdG in lung epithelial cells, which suggests an accumulation of oxidative damage. At this time point, the IR group also showed increased numbers of pro-SPC-positive cells, which suggests the transdifferentiation of lung epithelial cells to a mesenchymal-like phenotype. With GGA treatment, 8-OHdG expression and the number of pro-SPC-positive cells were significantly reduced (Fig. 2).

GGA suppresses radiation-induced EMT signals. Next, the effect of GGA treatmentEMT, which is key in radiation-induced lung injury was examined. Following IR, immunofluorescence demonstrated decreased expression of E-cadherin, which is an epithelial marker, and increased expression of vimentin, which is a mesenchymal marker in the lung tissue (Fig. 3A). GGA treatment significantly prevented radiation-induced EMT in the lung 6 months after IR. These results were confirmed by western blot analysis of the E-cadherin, vimentin and $\mathrm{N}$-cadherin protein levels in the lung tissue lysates (Fig. 3B).

HSP70 inhibits radiation-induced EMT in L132 human lung epithelial cells. GGA treatment upregulated HSP70 expression in L132 cells. Consistent with in vivo data, GGA treatment significantly inhibited radiation-induced EMT in L132 cells, as demonstrated by the preserved expression of the epithelial 

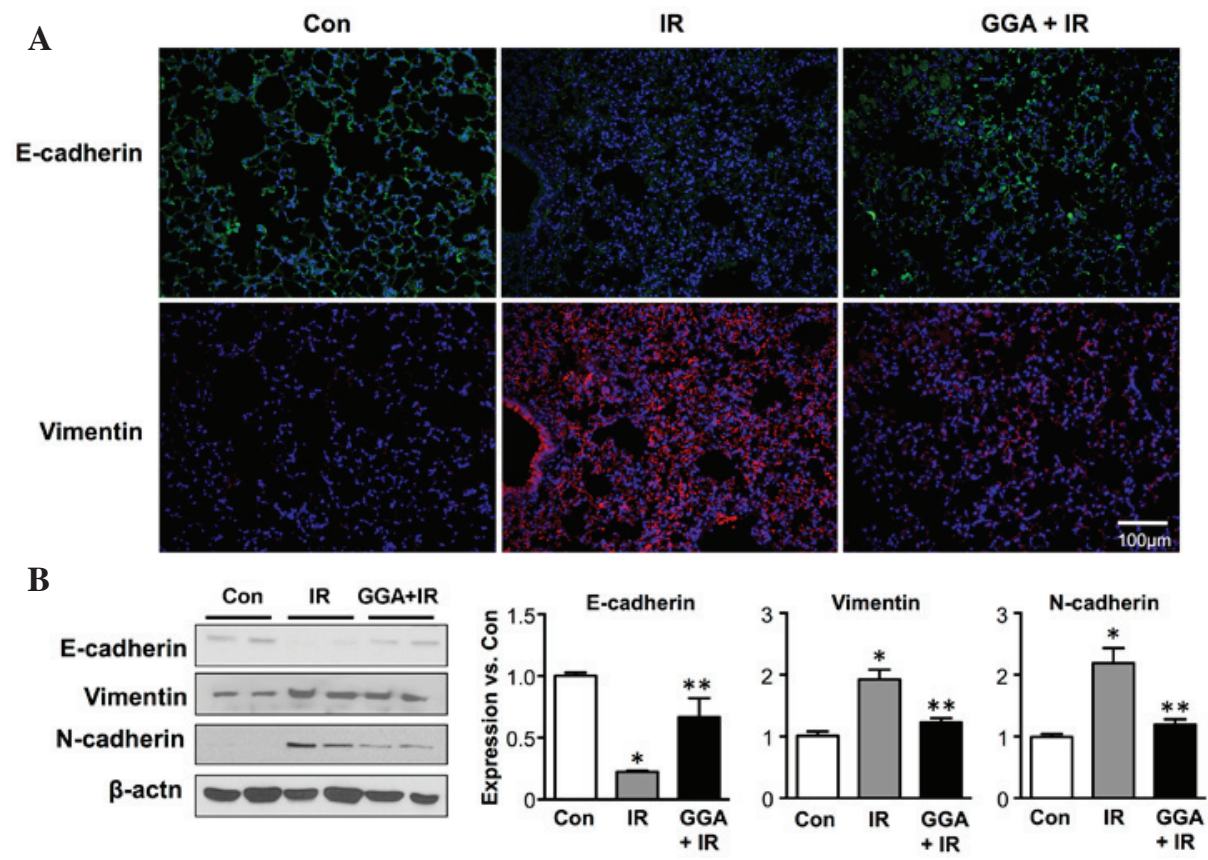

Figure 3. Evaluation of EMT marker expression in the lungs. (A) Representative images of E-cadherin and vimentin staining in the lungs 6 months after IR by immunofluorescence staining. (B) Quantification of the expression of E-cadherin and vimentin by western blotting ( $\mathrm{n}=3$ per group; " $\mathrm{P}<0.05$ vs. control, ${ }^{* *} \mathrm{P}<0.05$ vs. IR control). EMT, epithelial-to-mesenchymal transition; IR, irradiation; GGA, geranylgeranylacetone.
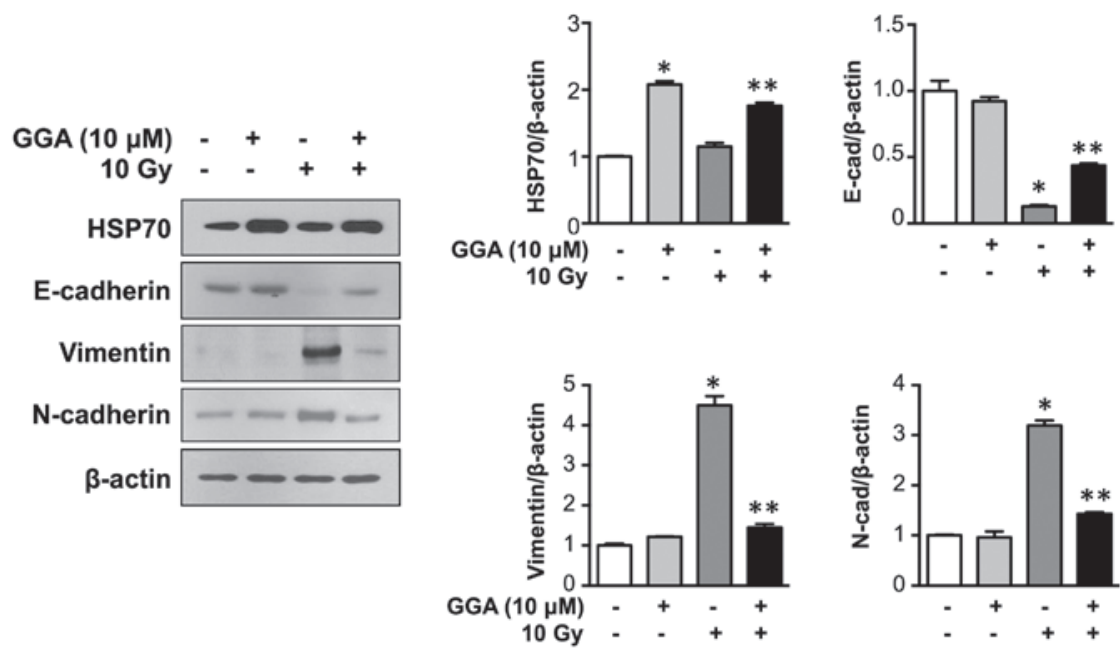

Figure 4. HSP70 expression prevents radiation-induced EMT marker expression in L132 human lung epithelial cells. Representative western blotting data for HSP70, E-cadherin, vimentin, and N-cadherin expression are shown. Expression of HSP70 was induced by GGA treatment in L132 cells. L132 cells were harvested 24 h after GGA treatment $(0$ to $15 \mu \mathrm{M})$ and subjected to western blotting, and the expression of HSP70, E-cadherin, vimentin, and $\mathrm{N}$-cadherin was quantified (n=3, $\mathrm{P}<0.05$ vs. $0 \mu \mathrm{M}$ control, ${ }^{* *} \mathrm{P}<0.05$ vs. IR control). GGA, geranylgeranylacetone; IR, irradiation; EMT, epithelial-to-mesenchymal transition; HSP70, heat shock protein 70 .

marker E-cadherin and the reduced upregulation of mesenchymal markers (vimentin and $\mathrm{N}$-cadherin) $(\mathrm{P}<0.05$, Fig. 4).

\section{Discussion}

In this study, it was demonstrated that increased expression of HSP70 in the lung during IR may prevent late signs of RILI, such as fibrosis. The mice were treated with orally with GGA $(200 \mathrm{mg} / \mathrm{kg}$ ) during the early stages of radiation injury (24 and $1 \mathrm{~h}$ ) prior to IR and 24, 48 and $72 \mathrm{~h}$ after IR. Increased expression of HSP70 was also detected in the lungs $24 \mathrm{~h}$ after oral GGA administration by western blotting (data not shown). GGA treatment resulted in decreased expression of EMT markers and attenuation of fibrosis in the lungs of mice treated with IR. Based on these results, inhibiting EMT during the acute phase of radiation exposure was important for the prevention of RILI.

Radiation-induced cell death is caused by direct DNA damage and indirect oxidative stress during the acute phase of radiation. However, late complications of radiation, such as fibrosis, are progressive and increase the complexity of the process involved in permanent epithelial cell injury (4). It was demonstrated that GGA significantly alleviates EMT marker expression and fibrosis in mouse lungs at the 6 months following IR. GGA is an inducer of HSP70, which protects cells from various stimuli, such as oxidative stress. GGA treatment markedly reduced the accumulation of oxidative damage by IR, which 
was shown by decreased expression of 8-OHdG. Moreover, Zhang et al (18) demonstrated that HSP70 protects lung injury from lethal oxidative stress. GGA treatment in mice decreased the expression of EMT markers pro-SPC and vimentin in mouse lung tissue. Radiation-induced injury of the lungs leads to expression of vimentin, a mesenchymal cell marker in lung epithelial cells (9). Additionally, the presence of pro-SPC-positive cells with an expanded interstitium suggests that the lung epithelial cells become detached from the basement membrane and migrate along with the extracellular matrix (19). Therefore, cells displaying an EMT phenotype express pro-SPC, which is consistent with the loss of epithelial marker expression. These results suggest that GGA could alleviate radiation-induced lung fibrosis in mouse lungs by preventing EMT marker expression.

As alveolar epithelial cells perform a critical function as a barrier against infections as well as in the inflammatory response, severe injury of the respiratory epithelium promotes the fibrotic process (20). GGA-treated lung tissue showed significantly higher expression of E-cadherin protein when compared with the IR groups. E-cadherin is a calcium-dependent adhesion molecule expressed on the surface of epithelial cells (21). Normal expression and functional activity of E-cadherin is critical for the maintenance of tight junctions between epithelial cells and for the maintenance of normal function of the paracellular barrier in the airway epithelia (9). It also confirmed that the overexpression of HSP70 following GGA treatment during IR, effectively inhibited the expression of mesenchymal-like phenotypes and GGA prevented the IR-induced loss of E-cadherin in vitro. The results from the current study suggest that GGA directly prevents EMT and indirectly protects lung epithelial cells.

The present study demonstrates that a protective effect of GGA on RILI is associated with the inhibition of EMT, which prevents late fibrosis. Moreover, increased expression of HSP70 by oral administration of GGA before radiation and during the acute phase of radiation injury effectively inhibited EMT marker expression, which appears to be important for the prevention of lung fibrosis. These results suggest that an HSP70 inducer, such as GGA, could act as a mitigator or protective agent against RILI. The current study demonstrated that oral administration of GGA, a non toxic HSP70-inducing anti-ulcer drug, effectively protects normal lung tissue against radiation-induced injury. GGA may be a promising therapeutic target of lung fibrosis subsequent to radiotherapy. However, further studies are required to clarify the influence of GGA on cancer treatment.

\section{Acknowledgements}

The current study was supported by the National Research Foundation (NRF) and Ministry of Science, ICT and Future Planning, Korean Government, through its National Nuclear Technology Program (grant nos. NRF-2014M2A2A7044825 and NRF-2013M2A2A7043580).

The English in this document has been checked by at least two professional editors from American Journal Experts.

\section{References}

1. McDonald S, Rubin P, Phillips TL and Marks LB: Injury to the lung from cancer therapy: Clinical syndromes, measurable endpoints and potential scoring systems. Int J Radiat Oncol Biol Phys 31: 1187-1203, 1995.
2. Robnett TJ, Machtay M, Vines EF, McKenna MG, Algazy KM and McKenna WG: Factors predicting severe radiation pneumonitis in patients receiving definitive chemoradiation for lung cancer. Int J Radiat Oncol Biol Phys 48: 89-94, 2000.

3. Hughes-Davies L, Tarbell NJ, Coleman CN, Silver B Shulman LN, Linggood R, Canellos GP and Mauch PM: Stage IA-IIB Hodgkin's disease: Management and outcome of extensive thoracic involvement. Int J Radiat Oncol Biol Phys 39: 361-369, 1997.

4. Cappuccini F, Eldh T, Bruder D, Gereke M, Jastrow H, Schulze-Osthoff K, Fischer U, Köhler D, Stuschke M and Jendrossek V: New insights into the molecular pathology of radiation-induced pneumopathy. Radiother Oncol 101: 86-92, 2011.

5. Wynn TA: Integrating mechanisms of pulmonary fibrosis. J Exp Med 208: 1339-1350, 2011.

6. Balli D, Ustiyan V, Zhang Y, Wang IC, Masino AJ, Ren X, Whitsett JA, Kalinichenko VV and Kalin TV: Foxm1 transcription factor is required for lung fibrosis and epithelial-to-mesenchymal transition. EMBO J 32: 231-244, 2013.

7. Pozharskaya V, Torres-González E, Rojas M, Gal A, Amin M, Dollard S, Roman J, Stecenko AA and Mora AL: Twist: A regulator of epithelial-mesenchymal transition in lung fibrosis. PLoS One 4: e7559-e7510, 2009.

8. Hodge S, Holmes M, Banerjee B, Musk M, Kicic A, Waterer G, Reynolds PN, Hodge G and Chambers DC: Posttransplant bronchiolitis obliterans syndrome is associated with bronchial epithelial to mesenchymal transition. Am J Transplant 9: 727-733, 2009.

9. Almeida C, Nagarajan D, Tian J, Leal SW, Wheeler K, Munley M, Blackstock $\mathrm{W}$ and Zhao $\mathrm{W}$ : The role of alveolar epithelium in radiation-induced lung injury. PLoS One 8: e53628, 2013.

10. Mao H, Li Z, Zhou Y, Li Z, Zhuang S, An X, Zhang B, Chen W, Nie J, Wang Z, et al: HSP72 attenuates renal tubular cell apoptosis and interstitial fibrosis in obstructive nephropathy. Am J Physiol Renal Physiol 295: F202-F214, 2008.

11. Tanaka K, Tanaka Y, Namba T, Azuma A and Mizushima T: Heat shock protein 70 protects against bleomycin-induced pulmonary fibrosis in mice. Biochem Pharmacol 80: 920-931, 2010.

12. Hagiwara S, Iwasaka H, Matsumoto S, Noguchi $T$ and Yoshioka H: Association between heat stress protein 70 induction and decreased pulmonary fibrosis in an animal model of acute lung injury. Lung 185: 287-293, 2007.

13. Niu P, Liu L, Gong Z, Tan H, Wang F, Yuan J, Feng Y, Wei Q, Tanguay RM and Wu T: Overexpressed heat shock protein 70 protects cells against DNA damage caused by ultraviolet $\mathrm{C}$ in a dose-dependent manner. Cell Stress Chaperones 11: 162-169, 2006.

14. Kawana K, Miyamoto Y, Tanonaka K, Han-no Y, Yoshida H, Takahashi M and Takeo S: Cytoprotective mechanism of heat shock protein 70 against hypoxia/reoxygenation injury. J Mol Cell Cardiol 32: 2229-2237, 2000.

15. Hirakawa $T$, Rokutan $K$, Nikawa $T$ and Kishi $K$ : Geranylgeranylacetone induces heat shock proteins in cultured guinea pig gastric mucosal cells and rat gastric mucosa. Gastroenterology 111: 345-357, 1996.

16. Niwa Y, Nakamura M, Miyahara R, Ohmiya N, Watanabe O, Ando $\mathrm{T}$, Kawashima $\mathrm{H}$, Itoh A, Hirooka $\mathrm{Y}$ and Goto $\mathrm{H}$ : Geranylgeranylacetone protects against diclofenac-induced gastric and small intestinal mucosal injuries in healthy subjects: A prospective randomized placebo-controlled double-blind cross-over study. Digestion 80: 260-266, 2009.

17. Liu J, Bao J, Hao J Peng Y and Hong F: HSP70 inhibits high glucose-induced Smad3 activation and attenuates epithelial-to-mesenchymal transition of peritoneal mesothelial cells. Mol Med Rep 10: 1089-1095, 2014.

18. Zhang Y, Zhang X, Shan P, Hunt CR, Pandita TK and Lee PJ: A protective Hsp70-TLR4 pathway in lethal oxidant lung injury. J Immunol 191: 1393-1403, 2013.

19. Kim KK, Kugler MC, Wolters PJ, Robillard L, Galvez MG, Brumwell AN, Sheppard D and Chapman HA: Alveolar epithelial cell mesenchymal transition develops in vivo during pulmonary fibrosis and is regulated by the extracellular matrix. Proc Natl Acad Sci USA 103: 13180-13185, 2006.

20. Adamson IY, Young L and Bowden DH: Relationship of alveolar epithelial injury and repair to the induction of pulmonary fibrosis. Am J Pathol 130: 377-383, 1988.

21. Takeichi M: Cadherin cell adhesion receptors as a morphogenetic regulator. Science 251: 1451-1455, 1991. 